\title{
A Model of Anatomically Opposed Ischaemia: Revisited
}

\author{
Peter R Johnston \\ Griffith University, Brisbane, Australia
}

\begin{abstract}
This study aims to gain further understanding of anatomically opposed ischaemia, or "ischaemic STsegment counterpoise”, by simulating body surface potential distributions resulting from two regions of partial thikcness ischaemia in the left ventricle during the STsegment.

The finite volume method was used to solve the passive bidomain equation in a torso with an idealised model of the heart. Regions of ischaemia of varying size were placed in various positions in the anterior and posterior regions of the ventricular wall.

Simulations show that the sources associated with the anterior ischaemic region dominated the body surface potential distribution, irrespective of the relative sizes of the two ischaemic regions. However, further modelling is required to establish a theoretical basis to understand ischaemic ST-segment counterpoise.
\end{abstract}

\section{Introduction}

Ischaemic ST-segment counterpoise is a phenomenon that arises when anatomically opposed patches of subendocardial ischaemia are present in the human heart. This phenomenon is believed to cause a false negative outcome in an exercise stress test [1-3]. The rationale for this phenomenon is based on a patient study where both severe left anterior descending and right coronary artery stenoses produced severe myocardial infarction (demonstrated by thallium-210 positron emission computerised tomography imaging), but produced a normal exercise electrocardiogram. One possible explanation for this observation is that there exist equal sized ischaemic regions on opposite sides of the heart, which lead to a net cancellation of the resulting ST-segment vectors during an ischaemic episode [2].

In an attempt to explain this phenomenon, a previous study [4] considered an isolated left ventricle in the shape of a half ellipsoid and considered the effect of two regions of subendocardial ischaemia included in the ventricular wall, placed at varying positions within the tissue. These regions were also of varying size, ranging from being equal to one another to one being significantly larger than the other. The conclusion reached was that one large anterior and one small posterior regions of ischaemia could yield an epicardial potential distribution (EPD) that was not significantly different to one large anterior region. However, having two ischaemic regions of similar size was identifiable on the EPD.

The present study consists of an idealised heart with right and left ventricles placed inside a realistic torso. This is closer to reality than the previous study. Each simulation considered here contains two regions of ischaemia of varying sizes and the results are discussed in terms of the resulting body surface potential distributions (BSPD).

\section{Methods}

For this study it was assumed that the thorax consisted of a heart with left and right ventricular walls, separated by a septum set within a homogeneous torso. The electric potential within the cardiac tissue was assumed to be governed by the passive bidomain equation

$$
\nabla \cdot\left(\mathbf{M}_{\mathbf{i}}+\mathbf{M}_{\mathbf{e}}\right) \nabla \phi_{e}=-\nabla \cdot \mathbf{M}_{\mathbf{i}} \nabla \phi_{\mathbf{m}}
$$

and by Laplace's equation in the remainder of the torso

$$
\nabla^{2} \phi_{b}=0
$$

which was assumed to be filled with blood. In these equations, $\phi_{e}$ is the extracellular potential in the cardiac tissue, $\phi_{m}$ is the specified transmembrane potential distribution and $\phi_{b}$ is the potential in the remainder of the body. The matrices $\mathbf{M}_{\mathbf{i}}$ and $\mathbf{M}_{\mathbf{e}}$ represent conductivity tensors for the intracellular $(i)$ and extracellular $(e)$ spaces, respectively.

The conductivity tensors take the form [5]

$$
\mathbf{M}_{\mathbf{q}}=\mathbf{A G}_{\mathbf{q}} \mathbf{A}^{\mathrm{T}}
$$

for $q=i$ or $e$, where $\mathbf{G}_{\mathbf{q}}$ is a diagonal matrix containing the conductivity values along the fibre direction $\left(g_{q l}\right)$, across the fibre sheets $\left(g_{q t}\right)$ and normal to the sheets of fibres $\left(g_{q n}\right)$. Cardiac tissue fibres were generated using a rule based model [6], where it was assumed that the fibres rotated through $120^{\circ}$ from the endocardium (at an angle of $45^{\circ}$ to the plane of the base of the ventricles) to the epicardium (at an angle of $-75^{\circ}$ ). Conductivity values used in 


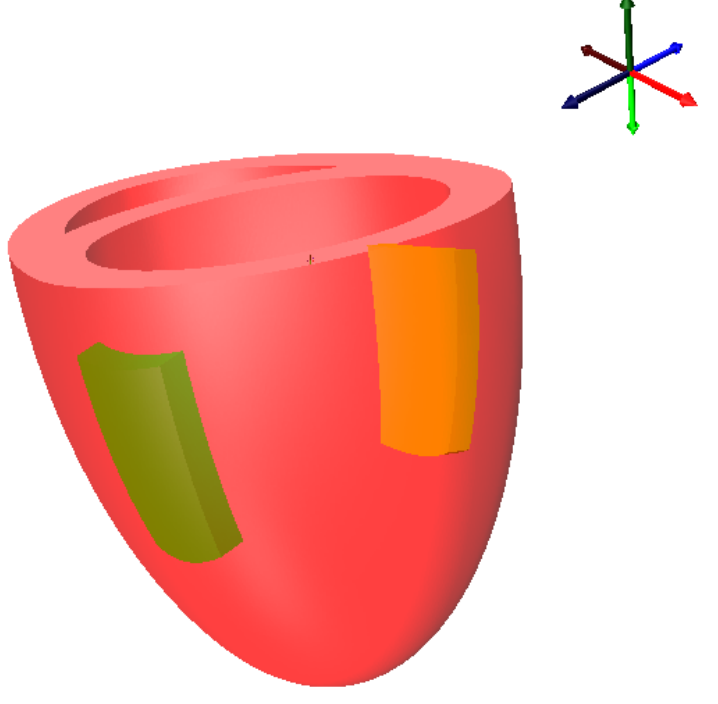

Figure 1. Model of the heart showing the large anterior and posterior ischaemic regions corresponding to scenario 1.

this study were $g_{i l}=2.4, g_{e l}=2.4, g_{i t}=0.35, g_{e t}=2.0$, $g_{i n}=0.08$ and $g_{e n}=1.1$, all with units of $\mathrm{mS} / \mathrm{cm}$ [7]. Finally, the conductivity of blood was set to $g_{b}=6.7 \mathrm{mS} / \mathrm{cm}$ [8].

Boundary conditions for the electrical potential were that the outer torso was insulated, there was no current flow from the intracellular space in the tissue into the blood and there was continuity of potential and current between the extracellular tissue space and the blood.

The heart itself was created by merging a half ellipsoid to create the left ventricle and appending another quarter of an ellipsoid to create the right ventricle. Overall the left ventricle was $8 \mathrm{~cm}$ high with an outer radius of $4 \mathrm{~cm}$ and the right ventricle was also $8 \mathrm{~cm}$ high with an outer radius of $8 \mathrm{~cm}$ (Figure 1). Throughout the heart, the tissue was uniformly $1 \mathrm{~cm}$ thick. This heart was placed inside a realistically shaped torso [9], with its centre of gravity moved $3 \mathrm{~cm}$ to the right and $4.125 \mathrm{~cm}$ forward from the centre of gravity of the torso. Both the geometry of the heart and the creation of the mesh were carried out using the open source mesh generator gmsh [10] and resulted in an overall mesh consisting of approximately 680,000 nodes and 4,150,000 tetrahedral elements, with approximately 220,000 nodes and 1,220,000 tetrahedral elements in the heart tissue. Note that the number of nodes and elements varied between scenarios due to the different ischaemic region configurations considered. The heart-torso geometry used in this study is shown in Figure 2.

Ischaemia was included in the tissue in a regular block type fashion. All regions of ischaemia extended through

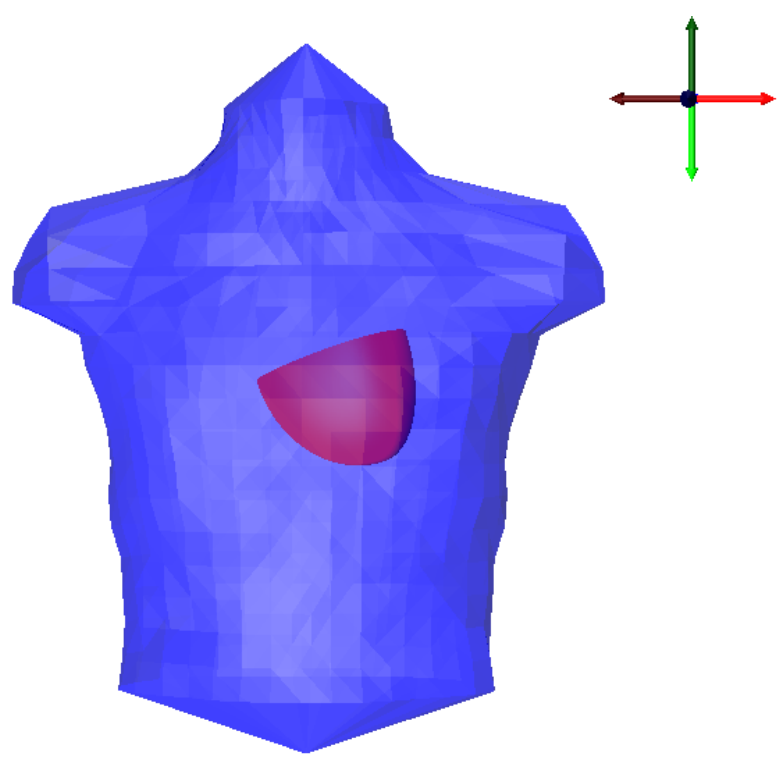

Figure 2. Model of the heart-torso system showing the relative size, position and orientation of the heart.

$50 \%$ of the thickness of the tissue, starting at $20 \%$, extending to $70 \%$. One reason for choosing such a simple model for the heart geometry was to enable easy control of the placement of the ischaemic regions. Each region is defined by an angular range in the polar $(\phi)$ and azimuthal $(\theta$, measured from the base of the heart) directions. These are summarised in Table 1, with Figure 1 showing scenario 2 with large anterior and posterior regions of ischaemia, as an example.

The overall governing equations are solved using an electrocardiography dedicated implementation [11] the finite volume method.

\section{Results}

Table 2 summarises the ranges of the body surface and heart surface potential found from the six different scenarios listed in Table 1. From this it would appear that the effect of the anterior ischaemic region dominates the BSPD. The presence of the posterior ischaemic region causes the potential distribution to become more negative (compare scenario 1 with scenarios 2 and 3 ) with the size of the anterior region affecting the size of this shift (compare scenario 2 with scenario 3 , and scenario 4 with scenario 5). When the size of the anterior ischaemic region is reduced (scenarios 4 and 5), the range of the potentials decreases, even when there is a large anterior ischaemic region. Note the small range of body surface potentials for the posterior ischaemic region (scenario 6). One further observation from this is that the range of epicardial potentials is reasonably 


\begin{tabular}{|c|c|c|c|c|c|c|c|c|l|}
\hline Scenario & $\theta_{l}^{(a)}$ & $\theta_{u}^{(a)}$ & $\phi_{l}^{(a)}$ & $\phi_{u}^{(a)}$ & $\theta_{l}^{(p)}$ & $\theta_{u}^{(p)}$ & $\phi_{l}^{(p)}$ & $\phi_{u}^{(p)}$ & Comments \\
\hline 1 & 10 & 40 & -40 & 0 & - & - & - & - & Baseline Anterior \\
2 & 10 & 40 & -40 & 0 & 10 & 40 & 140 & 180 & Large Anterior, Large Posterior \\
3 & 10 & 40 & -40 & 0 & 30 & 50 & 145 & 175 & Large Anterior, Small Posterior \\
4 & 30 & 50 & -35 & -5 & 10 & 40 & 140 & 180 & Small Anterior, Large Posterior \\
5 & 30 & 50 & -35 & -5 & 30 & 50 & 145 & 175 & Small Anterior, Small Posterior \\
6 & - & - & - & - & 10 & 40 & 140 & 180 & Baseline Posterior \\
\hline
\end{tabular}

Table 1. Summary of the ischaemic regions considered in this study. The regions are defined in terms of the upper $(u)$ and lower $(l)$ values for the polar $(\phi)$ and azimuthal $(\theta)$ angles, as well as the extent in the transmural direction, given as a percentage of the thickness of the ventricular wall. The superscripts $(a)$ and $(p)$ refer to the anterior and posterior ischaemic regions, respectively.

consistent across all scenarios.

Figure 3 shows the BSPD and EPD (in $\mathrm{mV}$ ) for scenario 1 , a single anterior region of ischaemia, both relative to a Wilson central terminal. On the body surface there is a region of positive potential just to the left of the centre of the torso, with two regions of negative potential adjoining the central region. This pattern is similar to that seen on the heart surface. Note that the zero potential line is continuous around the body and weaves its way between the maximum and the two minima.

Figure 4 shows the BSPD and the EPD for scenario 2, consisting of two large regions of ischaemia, both anterior and posterior. Here the BSPD is similar to that in Figure 3, with the main difference being that the zero contour is now in two parts, with regions of negative potential surrounding the entire positive region. Interestingly, the range of epicardial potentials is wider in this scenario than for scenario 1 , but the range of potential values on the body surface has become more negative. The BSPD in this figure is typical of the BSPDs in scenarios 3, 4 and 5, except that in scenarios 4 and 5, the anterior regions of ischaemia are smaller and the range of body surface potentials is reduced.

\section{Discussion}

From the results presented in Table 2 and Figures 3 and Figures 4 it would appear that for two diametrically op-

\begin{tabular}{|c|c|c|c|c|}
\hline Scenario & Min. BS & Max. BS & Min. HS & Max. HS \\
\hline 1 & -0.039 & 0.18 & -0.64 & 1.3 \\
2 & -0.068 & 0.16 & -0.94 & 1.5 \\
3 & -0.055 & 0.17 & -0.68 & 1.6 \\
4 & -0.047 & 0.040 & -0.94 & 1.5 \\
5 & -0.035 & 0.049 & -0.59 & 1.6 \\
6 & -0.016 & 0.0055 & -0.89 & 1.4 \\
\hline
\end{tabular}

Table 2. Minima and maxima for the body surface (BS) and epicardial (HS) potential distributions (in $\mathrm{mV}$ ) for the different scenarios.

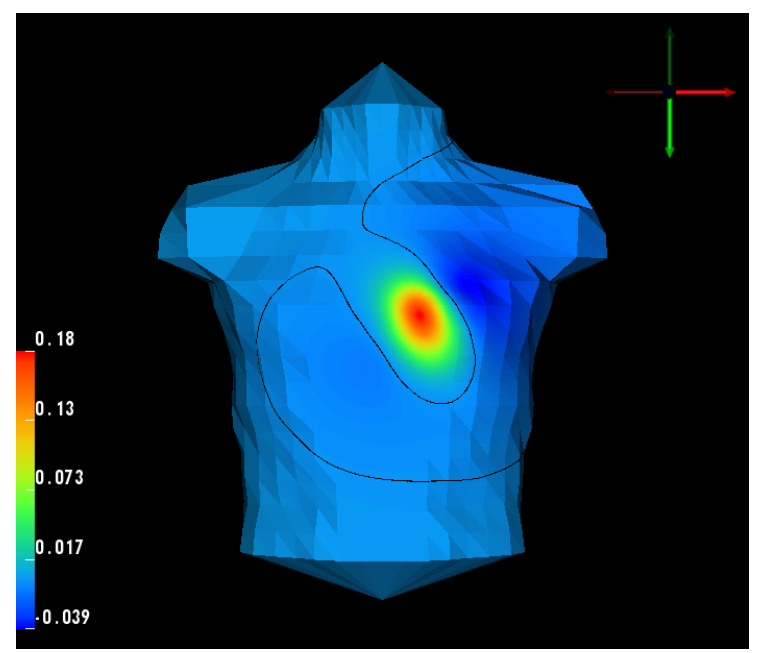

(a) Body surface potential distribution.

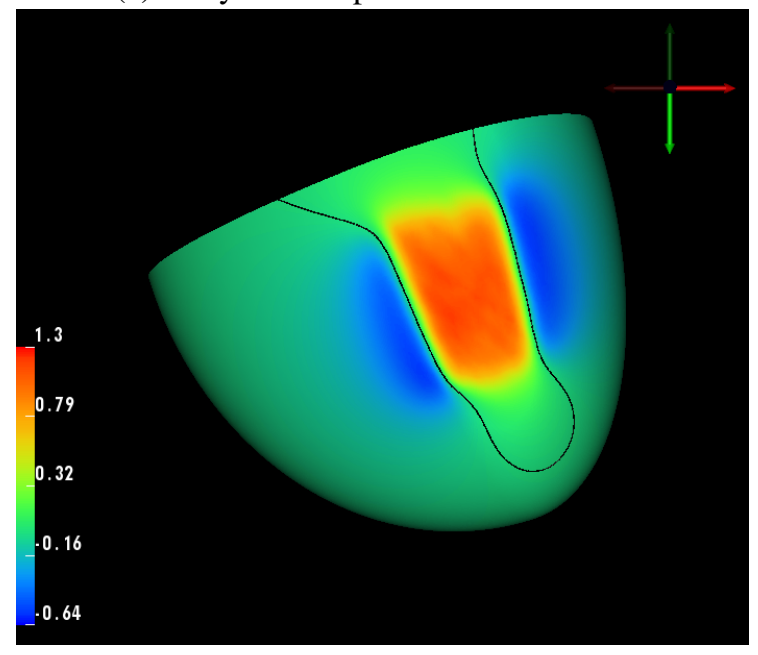

(b) Epicardial potential distribution.

Figure 3. Potential distributions for scenario 1, with a single large anterior ischaemic region. The solid black line in the plots is the zero potential line. 


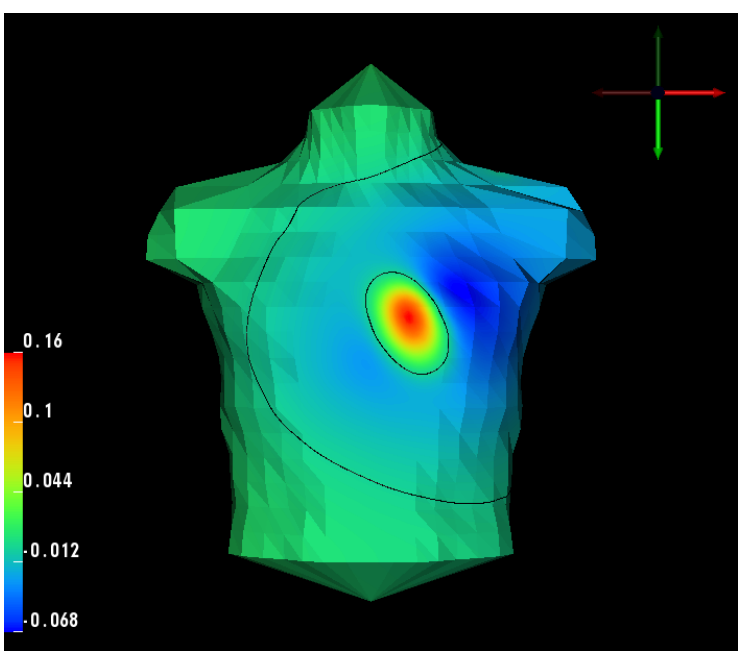

(a) Body surface potential distribution.

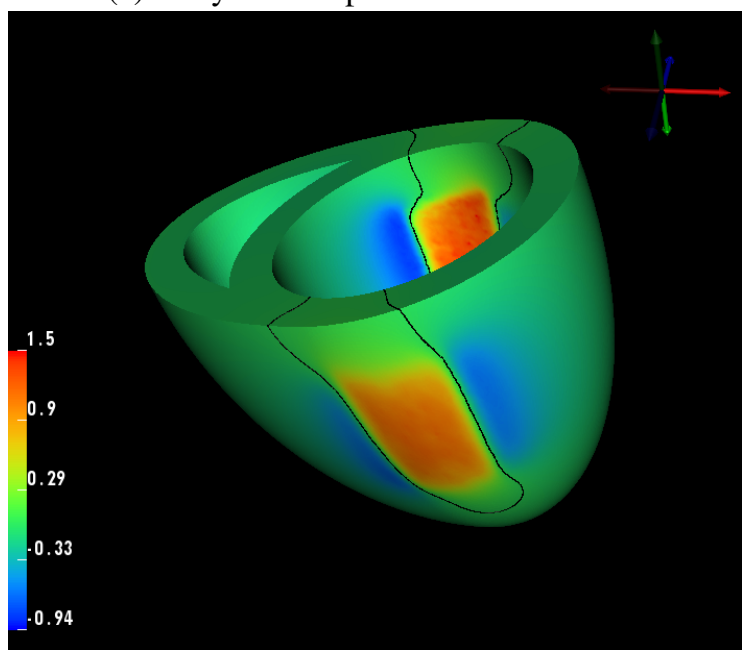

(b) Epicardial potential distribution.

Figure 4. Potential distributions for scenario 2, with large anterior and posterior ischaemic regions.

posed regions of ischaemia, the anterior region dominates the posterior region in terms of the effect on the BSPD. This is even in the case when there is a large posterior region of ischaemia and a small anterior region. The most likely explanation for this is the fact that the anterior region is much closer to the body surface and there is less attenuation of its effect, compared to the posterior region.

Therefore, based on these observations, it appears that the possibility that two diametrically opposed regions of ischaemia could cancel each other out on the body surface is unlikely.

Having said that, the present study has some limitations. Perhaps if the study were carried out in a transient manner, by solving the active bidomain equation, then the observation might be different. Also, the fact that the model here only consisted of a heart and torso, with no other internal organs, with the main conductivity set to that of blood, might change the results. Hence, further modelling work is required to establish, from a mathematical and simulation point of view, whether the two diametrically opposed regions of ischaemia cancel each other out.

\section{References}

[1] Madias JE. Ischaemic ST-segment counterpoise: One mechanism of false electrocardiographic response to exercise stress testing. American Jounral of Noninvasive Cardiology 1994;8:194-199.

[2] Madias JE, Mahjoub M, Valance J. The paradox of negative exercise stress ECG/positive thallium scintigram: ischemic ST-segment counterpoise as the underlying mechanism. Journal of Electrocardiology 1996;29(3):243-248.

[3] Madias JE, Khan M, Manyam B. The role of "ischemic ST-segment counterpoise" in rendering the response of exercise electrocardiogram falsely negative. Clinical Cardiology 1997;20:489-492.

[4] Johnston PR. A model of anatomically opposed ischaemia. In Murray A (ed.), Computing in Cardiology, volume 39. IEEE, IEEE Press, September 2012; 465-468.

[5] Gulrajani RM. Bioelectricity and Biomagnetism. New York: John Wiley and Sons, 1998.

[6] Bayer JD, Blake RC, Plank G, Trayanova NA. A novel rulebased algorithm for assigning myocardial fiber orientation to computational heart models. Annals of Biomedical Engineering 2012;40(10):2243-2254.

[7] Johnston BM. Six conductivity values to use in the bidomain model of cardiac tissue. IEEE Transactions on Biomedical Engineering 2016;63(7):1525-1531.

[8] Rush S, Abildskov JA, McFee R. Resistivity of body tissues at low frequencies. Circulation Research 1963;12:40-50.

[9] Horacek BM. Numerical model of an inhomogeneous human torso. Advances in Cardiology 1974;10:51-57.

[10] Geuzaine C, Remacle JF. Gmsh: a three-dimensional finite element mesh generator with built-in pre- and postprocessing facilities. International Journal for Numerical Methods in Engineering 2009;79(11):1309-1331.

[11] Johnston PR. A finite volume method solution for the bidomain equations and their application to modelling cardiac ischaemia. Computer Methods in Biomechanics and Biomedical Engineering 2010;13(2):157-170.

\section{Address for correspondence:}

Peter Johnston, School of Environment and Science and Queensland Micro- and Nanotechnology Centre, Griffith University, Nathan, Queensland, Australia, 4111, p.johnston@ griffith.edu.au 